\title{
Design of Iterative Learning Controllers for Linear Discrete Systems with Multiple Time Delays
}

\author{
Hansheng Wu Member (Hiroshima Prefectural University, hansheng@pu-hiroshima.ac.jp)
}

Keywords: Discrete systems, time delays, iterative learning control, asymptotic tracking control.

In a number of practical control problems, there is a class of repetitive dynamical systems, such as robotic control systems, neuromuscular stimulation systems, and so on. For such repetitive dynamical systems, the so-called iterative learning control laws have been introduced. Generally speaking, by employing an iterative learning control algorithm, one can gradually improve or perfect the system performance of a specified task, based on the previous performances of the identical tasks.

It is well known that the main advantage of the iterative learning control strategy is to require less a priori knowledge about the system dynamics and less computational effort than many other types of control strategies. Therefore, the problem of iterative learning control for repetitive dynamical systems has received considerable attention, and many results have been obtained. In particular, there are some works in which an iterative learning control scheme has been applied to the analysis and design of continuous dynamical systems with time-delay. On the other hand, the iterative learning control problem of discrete dynamical systems is also considered in the control literature, and some methods are presented to deal with such a class of iterative learning control problems. However, few efforts are made to consider the problem of iterative learning control for discrete dynamical systems with time-delay. It seems that for discrete timedelay dynamical systems, the similar results have not been reported yet in the control literature.

In this paper, we consider a discrete-time dynamical system with time-delay, described by

$$
\begin{aligned}
x(m+1)= & A x(m)+B u(m) \\
& +\sum_{\mu=1}^{r} E_{\mu} x\left(m-h_{\mu}(m)\right) \\
y(m)= & C x(m) \ldots \ldots \ldots \ldots .
\end{aligned}
$$

where $x(m) \in R^{n}$ is the current value of the state, $u(m) \in$ $R^{m}$ is the control (or input) vector, $y(m) \in R^{l}$ is the output vector, $A, B, C$ are constant matrices of appropriate dimensions, and the matrix $E_{\mu}, \mu=1,2, \ldots, r$, represent the delayed state perturbations. Moreover, the time-varying delay $h_{\mu}(m)$ is assumed to be any bounded nonnegative integer function which is not required to be known for the system designer. That is, $0 \leq h_{\mu}(m) \leq \bar{h}_{\mu}$, where $\bar{h}_{\mu}$ is a positive integer.

The initial condition for the system with time delays is given by

$$
x(m)=\chi(m), \quad m \in \Gamma_{0}
$$

where $\chi(m)$ is a bounded function on $\Gamma_{0}$, and

$$
\Gamma_{0}:=\{-\bar{h},-\bar{h}+1,-\bar{h}+2, \ldots,-1,0\}
$$

and where $\bar{h}:=\max _{\mu}\left\{\bar{h}_{\mu}, \mu=1,2, \ldots, r\right\}$.

Here, the initial condition $\chi(m), m \in \Gamma_{0}$, for (1) is also assumed to be unknown at each iteration.

For discrete time-delay system (1), it is supposed that a desired output trajectory $y_{r}(m) \in R^{l}$ is given for a finite time interval $m \in \Gamma_{0}^{N}:=\{0,1,2, \ldots, N\}$. Then, the error between the desired output and the actual output trajectories of the discrete time-delay system can be represented by

$$
e(m)=y_{r}(m)-y(m) \ldots \ldots \ldots \ldots \ldots \ldots \ldots \ldots
$$

where $m \in \Gamma_{0}^{N}$ and $e(m) \in R^{l}$.

Here, we use the superscript $k$ to denote the iteration number of learning processes. Therefore, $x^{[k]}(m), u^{[k]}(m)$, $y^{[k]}(m)$ represent the corresponding vectors at the $k$ th iteration.

Now, the main objective of this paper is to find the iterative learning control laws for discrete time-delay system (1) with the unknown initial state such that the output error $e(m)$ between the given desired output $y_{r}(m)$ and the actual output $y(m)$ is identical for all $m \in \Gamma_{0}^{N}$, through the iterative learning process. That is, for all $m \in \Gamma_{0}^{N}$,

$$
\lim _{k \rightarrow \infty}\left\|e^{[k]}(m)\right\|=\lim _{k \rightarrow \infty}\left\|y_{r}(m)-y^{[k]}(m)\right\|=0
$$

For this purpose, we present a method whereby a class of iterative learning control schemes can be constructed for discrete time-delay systems. We also show that under some given conditions, the constructed iterative learning controllers can guarantee the asymptotic convergence of the output error between the given desired output and the actual output through the iterative learning process. Finally, an illustrative example is given to demonstrate the validity of the results obtained in the paper. 


\title{
Design of Iterative Learning Controllers for Linear Discrete Systems with Multiple Time Delays
}

\author{
Hansheng $\mathrm{Wu}^{*}$ Member
}

\begin{abstract}
The problem of iterative learning control is considered for a class of linear discrete systems with multiple time delays. In this paper, it is assumed that the initial condition for discrete time-delay systems is unknown at each iteration. For such a class of discrete time-delay systems, a method is presented whereby a class of discrete iterative learning control schemes can be constructed. It is shown that under some given conditions, the constructed iterative learning controllers can guarantee the asymptotic convergence of the output error between the given desired output and the actual output through the iterative learning process. Finally, a numerical example is given to demonstrate the validity of the results.
\end{abstract}

Keywords: Discrete systems, time delays, iterative learning control, asymptotic tracking control.

\section{Introduction}

In a number of practical control problems, there is a class of repetitive dynamical systems, such as robotic control systems, neuromuscular stimulation systems, and so on. For such repetitive dynamical systems, the so-called iterative learning control laws have been introduced in Ref. (1). Generally speaking, by employing an iterative learning control algorithm, one can gradually improve or perfect the system performance of a specified task, based on the previous performances of the identical tasks.

It is well known that the main advantage of the iterative learning control strategy is to require less a priori knowledge about the system dynamics and less computational effort than many other types of control strategies. Therefore, the problem of iterative learning control for repetitive dynamical systems has received considerable attention, and many results have been obtained (see, e.g., Refs. (2)-(9), and the references therein). In particular, there are some works in which an iterative learning control scheme has been applied to the analysis and design of continuous dynamical systems with time-delay. In Ref. (10), for example, the design of an iterative learning controller is considered for a class of linear systems with time delay, and an iterative learning control algorithm is proposed such that the output of the considered time-delay dynamical systems can track a given desired trajectory. In Ref. (11), a class of PID-type iterative learning control schemes is proposed for uncertain nonlinear systems with state delays, and the convergence conditions for the proposed high-order learning control are derived. It should be noted that, in Ref. (10) and Ref. (11), the initial conditions are as-

\footnotetext{
* Department of Information Science

Hiroshima Prefectural University

562 Nanatsuka-cho, Shobara-shi, Hiroshima 727-0023

Email: hansheng@pu-hiroshima.ac.jp
}

sumed to be known, and can be employed in each iteration. On the other hand, the iterative learning control problem of discrete dynamical systems is also considered in the control literature, and some results are obtained (see, e.g. Refs. (12)-(15), and the references therein). However, few efforts are made to consider the problem of iterative learning control for discrete dynamical systems with time-delay. It seems that for discrete time-delay systems, the similar results have not been reported yet in the control literature.

In this paper, we consider the problem of iterative learning control for linear discrete dynamical systems with time-delay. We assume that the initial condition for discrete time--delay systems is unknown at each iteration. For such a discrete time-delay system, we want to present a method whereby a class of iterative learning control schemes can be constructed. We also show that under some given conditions, the constructed iterative learning controllers can guarantee the asymptotic convergence of the output error between the given desired output and the actual output through the iterative learning process.

The paper is organized as follows. In Section 2, the iterative learning control problem to be tackled is stated. In Section 3, we propose a class of iterative learning control schemes for discrete time-delay systems. In Section 4 , a numerical example is given to illustrate the use of our results. The paper is concluded in Section 5 with a brief discussion of the results.

\section{Problem Formulation and Assumptions}

We consider a discrete-time dynamical system with time-delay, described by the following difference equations:

$$
\begin{aligned}
x(m+1)= & A x(m)+B u(m) \\
& +\sum_{\mu=1}^{r} E_{\mu} x\left(m-h_{\mu}(m)\right) \cdots \cdots \cdots
\end{aligned}
$$




$$
y(m)=C x(m)
$$

where $x(m) \in R^{n}$ is the current value of the state, $u(m) \in R^{m}$ is the control (or input) vector, $y(m) \in R^{l}$ is the output vector, $A, B, C$ are constant matrices of appropriate dimensions, and the matrix $E_{\mu}, \mu=1,2, \ldots, r$, represent the delayed state perturbations. Moreover, the time-varying delay $h_{\mu}(m)$ is assumed to be any bounded nonnegative integer function which is not required to be known for the system designer. That is, $0 \leq h_{\mu}(m) \leq \bar{h}_{\mu}$, where $\bar{h}_{\mu}$ is a positive integer.

The initial condition for the system with time delays is given by

$$
x(m)=\chi(m), \quad m \in \Gamma_{0}
$$

where $\chi(m)$ is a bounded function on $\Gamma_{0}$, and

$$
\Gamma_{0}:=\{-\bar{h},-\bar{h}+1,-\bar{h}+2, \ldots,-1,0\}
$$

and where

$$
\bar{h}:=\max _{\mu}\left\{\bar{h}_{\mu}, \mu=1,2, \ldots, r\right\}
$$

Here, the initial condition $\chi(m), m \in \Gamma_{0}$, for system (1) is assumed to be unknown at each iteration.

For system (1), it is supposed that a desired output trajectory $y_{r}(m) \in R^{l}$ is given for a finite time interval $m \in \Gamma_{0}^{N}:=\{0,1,2, \ldots, N\}$. Then, the error between the desired output and the actual output trajectories of the system can be represented by

$$
e(m)=y_{r}(m)-y(m)
$$

where $m \in \Gamma_{0}^{N}$ and $e(m) \in R^{l}$.

Throughout this paper, we use the superscript $k$ to denote the iteration number of learning. Therefore, $x^{[k]}(m), u^{[k]}(m), y^{[k]}(m)$ represent the corresponding vectors at the $k$ th iteration.

Now, the main objective of this paper is to find the iterative learning control laws for system (1) with the unknown initial state such that the output error $e(m)$ between the given desired output $y_{r}(m)$ and the actual output $y(m)$ is identical for all $m \in \Gamma_{0}^{N}$, through the iterative learning process. That is, for all $m \in \Gamma_{0}^{N}$,

$$
\lim _{k \rightarrow \infty}\left\|e^{[k]}(m)\right\|=\lim _{k \rightarrow \infty}\left\|y_{r}(m)-y^{[k]}(m)\right\|=0
$$

Before giving our iterative learning control laws, we introduce for system (1) the following standard assumptions.

Assumption 2.1 For system (1), the matrix $C B$ is full rank.

Remark 2.1 It is obvious that Assumption 2.1 is standard, and by this assumption we means that the existence of iterative learning control laws can be guaranteed, which will be known from the conditions derived in the next sections.

Throughout this paper, $\|M\|$ denotes any norm of a vector (matrix) $M$.

\section{Iterative Learning Controllers}

In this section, for the problem stated in Section 2, we propose an input updating law for iterative learning control as follows. That is, for any $k \in\{0,1,2, \cdots\}$,

$$
u^{[k+1]}(m)=u^{[k]}(m)+\Gamma\left[e^{[k]}(m+1)-e^{[k]}(m)\right]
$$

where $m \in \Gamma_{0}^{N}$, and $\Gamma \in R^{m \times l}$ is an iterative learning control gain matrix which will be determined later, together with a learning algorithm on the initial condition, described by

$$
\chi^{[k+1]}(m)=\chi^{[k]}(m)+B \Gamma e^{[k]}(0), \quad m \in \Gamma_{0} \cdots
$$

where $u_{i}^{[0]}(m), m \in \Gamma_{0}^{N}$, is an arbitrary initial control input, and $\chi^{[0]}(m), m \in \Gamma_{0}$, is an arbitrary initial condition, which may be different from the unknown desired initial condition.

Then the following theorem can be obtained which shows that the iterative learning control laws described by (5) and (6) can guarantee the asymptotic convergence of the output error of discrete time-delay system (1).

Theorem 3.1 Consider the linear discrete timedelay systems described by (1) which satisfies Assumption 2.1. Given the desired output trajectory $y_{r}(m)$ over the finite time interval $\Gamma_{0}^{N}$, by employing the iterative learning control laws described by (5) and (6), the output error $e(m)$ of system (1) can be guaranteed to asymptotically converge to zero, i.e. for any $m \in \Gamma_{0}^{N}$,

$$
\lim _{k \rightarrow \infty}\left\|e^{[k]}(m)\right\|=\lim _{k \rightarrow \infty}\left\|y_{r}(m)-y^{[k]}(m)\right\|=0
$$

if there exists an iterative learning control gain matrix $\Gamma$ such that

$$
\|I-C B \Gamma\|<1
$$

where $I \in R^{l \times l}$ is an identity matrix.

Proof: For any given control input $u(m), m \in \Gamma_{0}^{N}$, the general solution $x(m)$ to discrete time-delay system (1) can be written in the following form:

$$
\begin{aligned}
x(m) & =A^{m} \chi(0)+\sum_{j=0}^{m-1} A^{m-j-1} B u(j) \\
& +\sum_{j=0}^{m-1} \sum_{\mu=1}^{r} A^{m-j-1} E_{\mu} x\left(j-h_{\mu}(j)\right) \cdots \cdots
\end{aligned}
$$

Thus, for the $k$ th iteration, it can be known from (9) that for any $m \in \Gamma_{0}^{N}$,

$$
\begin{gathered}
x^{[k]}(m)=A^{m} \chi^{[k]}(0)+\sum_{j=0}^{m-1} A^{m-j-1} B u^{[k]}(j) \\
+\sum_{j=0}^{m-1} \sum_{\mu=1}^{r} A^{m-j-1} E_{\mu} x^{[k]}\left(j-h_{\mu}(j)\right) \cdots \cdots
\end{gathered}
$$


Furthermore, by making use of (10) together with (5) and (6), the state error can be expressed as

$$
\begin{aligned}
& x^{[k+1]}(m)-x^{[k]}(m)=A^{m} B \Gamma e^{[k]}(0) \\
& +\sum_{j=0}^{m-1} A^{m-j-1} B \Gamma\left[e^{[k]}(j+1)-e^{[k]}(j)\right] \\
& +\sum_{j=0}^{m-1} \sum_{\mu=1}^{r} A^{m-j-1} E_{\mu} \\
& \quad \times\left(x^{[k+1]}\left(j-h_{\mu}(j)\right)-x^{[k]}\left(j-h_{\mu}(j)\right)\right)
\end{aligned}
$$

On the other hand, by making use of some trivial manipulations, we can obtain that for any $m \in \Gamma_{0}^{N}$,

$$
\begin{aligned}
\sum_{j=0}^{m-1} & A^{m-j-1} B \Gamma\left[e^{[k]}(j+1)-e^{[k]}(j)\right] \\
= & \sum_{t=1}^{m} A^{m-t} B \Gamma e^{[k]}(t)-\sum_{j=0}^{m-1} A^{m-j-1} B \Gamma e^{[k]}(j) \\
= & \sum_{t=0}^{m} A^{m-t} B \Gamma e^{[k]}(t)-A^{m} B \Gamma e^{[k]}(0) \\
= & -\sum_{j=0}^{m-1} A^{m-j-1} B \Gamma e^{[k]}(j) \\
= & \sum_{j=0}^{m-1} A^{m-j} B \Gamma e^{[k]}(j)+A^{0} B \Gamma e^{[k]}(m) \\
& -A^{m} B \Gamma e^{[k]}(0)-A^{-1} \sum_{j=0}^{m-1} A^{m-j} B \Gamma e^{[k]}(j) \\
= & B \Gamma e^{[k]}(m)-A^{m} B \Gamma e^{[k]}(0) \\
& +\left[I-A^{-1}\right] \sum_{j=0}^{m-1} A^{m-j} B \Gamma e^{[k]}(j)
\end{aligned}
$$

Then, the substitution of (12) into (11) yields

$$
\begin{aligned}
& x^{[k+1]}(m)-x^{[k]}(m)=B \Gamma e^{[k]}(m) \\
& +\left[I-A^{-1}\right] \sum_{j=0}^{m-1} A^{m-j} B \Gamma e^{[k]}(j) \\
& +\sum_{j=0}^{m-1} \sum_{\mu=1}^{r} A^{m-j-1} E_{\mu} \\
& \quad \times\left(x^{[k+1]}\left(j-h_{\mu}(j)\right)-x^{[k]}\left(j-h_{\mu}(j)\right)\right)
\end{aligned}
$$

Taking the norm of both sides of (13) and making use of the general properties of norms, we can obtain that for any $m \in \Gamma_{0}^{N}$,

$$
\begin{aligned}
& \left\|x^{[k+1]}(m)-x^{[k]}(m)\right\| \leq\|B \Gamma\|\left\|e^{[k]}(m)\right\| \\
& +\left\|I-A^{-1}\right\| \sum_{j=0}^{m-1}\left\|A^{m-j}\right\|\|B \Gamma\|\left\|e^{[k]}(j)\right\|
\end{aligned}
$$

$$
\begin{aligned}
& +\sum_{j=0}^{m-1} \sum_{\mu=1}^{r}\left\|A^{m-j}\right\|\left\|A^{-1}\right\|\left\|E_{\mu}\right\| \\
& \times\left\|x^{[k+1]}\left(j-h_{\mu}(j)\right)-x^{[k]}\left(j-h_{\mu}(j)\right)\right\|
\end{aligned}
$$

Moreover, letting

$$
\kappa:=\max \left\{\left\|A^{m-j}\right\|, m \in \Gamma_{0}^{N}, j \in \Gamma_{0}^{N}\right\}
$$

it follows from (14) that for any $m \in \Gamma_{0}^{N}$,

$$
\begin{aligned}
& \left\|x^{[k+1]}(m)-x^{[k]}(m)\right\| \leq\|B \Gamma\|\left\|e^{[k]}(m)\right\| \\
& +\kappa\left\|I-A^{-1}\right\| \sum_{j=0}^{m-1}\|B \Gamma\|\left\|e^{[k]}(j)\right\| \\
& +\kappa\left\|A^{-1}\right\| \sum_{j=0}^{m-1} \sum_{\mu=1}^{r}\left\|E_{\mu}\right\| \\
& \quad \times\left\|x^{[k+1]}\left(j-h_{\mu}(j)\right)-x^{[k]}\left(j-h_{\mu}(j)\right)\right\|
\end{aligned}
$$

By multiplying both sides of (15) by $a^{-\lambda m}$, where $a$ is the constant satisfying $a>1$ and $\lambda$ is any positive constant (i.e. $\lambda>0$ ), and by making use of some trivial manipulations, we can obtain that for any $m \in \Gamma_{0}^{N}$,

$$
\begin{aligned}
& \left\|x^{[k+1]}(m)-x^{[k]}(m)\right\| a^{-\lambda m} \\
& \leq\|B \Gamma\|\left\|e^{[k]}(m)\right\| a^{-\lambda m} \\
& +\kappa\left\|I-A^{-1}\right\| \sum_{j=0}^{m-1} a^{-\lambda(m-j)}\|B \Gamma\|\left\|e^{[k]}(j)\right\| a^{-\lambda j} \\
& +\kappa\left\|A^{-1}\right\| \sum_{j=0}^{m-1} \sum_{\mu=1}^{r} a^{-\lambda h_{\mu}(j)} a^{-\lambda(m-j)}\left\|E_{\mu}\right\| \\
& \times\left\|x^{[k+1]}\left(j-h_{\mu}(j)\right)-x^{[k]}\left(j-h_{\mu}(j)\right)\right\| \\
& \times a^{-\lambda\left(j-h_{\mu}(j)\right)}
\end{aligned}
$$

Letting

$$
\begin{aligned}
& \tilde{e}^{[k]}(m):=\max _{\rho}\left\{\left\|e^{[k]}(\rho)\right\| a^{-\lambda \rho}, \rho \in \Gamma_{\bar{h}}^{m}\right\} \\
& \tilde{z}^{[k]}(m):=\max _{\rho}\left\{\left\|x^{[k+1]}(\rho)-x^{[k]}(\rho)\right\| a^{-\lambda \rho}, \rho \in \Gamma_{\bar{h}}^{m}\right\}
\end{aligned}
$$

where $\Gamma_{\bar{h}}^{m}:=\{-\bar{h},-\bar{h}+1, \ldots,-1,0,1, \ldots, m\}$, and noting the fact that for any $a>1$ and $\lambda>0,0 \leq a^{-\lambda h_{\mu}(j)} \leq$ 1 , it follows from (16) that for any $m \in \Gamma_{0}^{N}$,

$$
\begin{aligned}
& \left\|x^{[k+1]}(m)-x^{[k]}(m)\right\| a^{-\lambda m} \\
& \leq\|B \Gamma\| \tilde{e}^{[k]}(m) \\
& \quad+\kappa\left\|I-A^{-1}\right\|\|B \Gamma\| \tilde{e}^{[k]}(m) \sum_{j=0}^{m-1} a^{-\lambda(m-j)}
\end{aligned}
$$




$$
\begin{aligned}
& +\kappa\left\|A^{-1}\right\| \tilde{z}^{[k]}(m) \sum_{j=0}^{m-1} a^{-\lambda(m-j)} \sum_{\mu=1}^{r}\left\|E_{\mu}\right\| \\
\leq & \|B \Gamma\| \tilde{e}^{[k]}(m) \\
& +\kappa\left\|I-A^{-1}\right\|\|B \Gamma\| \tilde{e}^{[k]}(m) \frac{a^{-\lambda}}{1-a^{-\lambda}} \\
& +\kappa\left\|A^{-1}\right\| \tilde{z}^{[k]}(m) \frac{a^{-\lambda}}{1-a^{-\lambda}} \sum_{\mu=1}^{r}\left\|E_{\mu}\right\| \\
= & \|B \Gamma\|\left(1+\frac{\kappa a^{-\lambda}}{1-a^{-\lambda}}\left\|I-A^{-1}\right\|\right) \tilde{e}^{[k]}(m) \\
& +\frac{\kappa \delta a^{-\lambda}}{1-a^{-\lambda}}\left\|A^{-1}\right\| \tilde{z}^{[k]}(m) \cdots \ldots . . .
\end{aligned}
$$

where

$$
\delta:=\sum_{\mu=1}^{r}\left\|E_{\mu}\right\|
$$

First of all, notice such a fact that for any real function $F(k)$ and for any nondecreasing real function $G(k)$,

$$
F(k) \leq G(k), \quad \forall k \in \Gamma_{\sigma}^{T}
$$

implies that for any $k \in \Gamma_{\sigma}^{T}$,

$$
\tilde{F}(k):=\max _{\rho}\left\{F(\rho): \rho \in \Gamma_{\sigma}^{k}, k \leq T\right\} \leq G(k)
$$

where $\Gamma_{\sigma}^{T}:=\{\sigma+1, \sigma+2, \ldots, T\}, \sigma$ and $T$ are any integral constants, and $\sigma \leq T$.

Now, it is obvious from the definitions that the functions $\tilde{e}^{[k]}(m)$ and $\tilde{z}^{[k]}(m)$ are nondecreasing on $\Gamma_{0}^{N}$. It follows that the right-hand side of inequality (17) is also nondecreasing. Therefore, in the light of the definitions of the functions $\tilde{e}^{[k]}(m)$ and $\tilde{z}^{[k]}(m)$ and the fact stated above, we find from (17) that for any $m \in \Gamma_{0}^{N}$,

$$
\begin{aligned}
\tilde{z}^{[k]}(m) \leq & \|B \Gamma\|\left(1+\frac{\kappa a^{-\lambda}}{1-a^{-\lambda}}\left\|I-A^{-1}\right\|\right) \tilde{e}^{[k]}(m) \\
& +\frac{\kappa \delta a^{-\lambda}}{1-a^{-\lambda}}\left\|A^{-1}\right\| \tilde{z}^{[k]}(m) \cdots \cdots(18)
\end{aligned}
$$

where $\lambda$ is any positive constant. Moreover, letting $\lambda$ be chosen such that

$$
1-\frac{\kappa \delta a^{-\lambda}}{1-a^{-\lambda}}\left\|A^{-1}\right\|>0
$$

Then, we can find from (18) that for any $m \in \Gamma_{0}^{N}$,

$$
\tilde{z}^{[k]}(m) \leq \frac{1-a^{-\lambda}+\kappa a^{-\lambda}\left\|I-A^{-1}\right\|}{1-a^{-\lambda}-\kappa \delta a^{-\lambda}\left\|A^{-1}\right\|}\|B \Gamma\| \tilde{e}^{[k]}(m)
$$

On the other hand, in the light of the definition of the output error $e(t)$ and (13), we can have that for any $m \in \Gamma_{0}^{N}$,

$$
e^{[k+1]}(m)=(I-C B \Gamma) e^{[k]}(m)
$$

$$
\begin{aligned}
& -C\left(I-A^{-1}\right) \sum_{j=0}^{m-1} A^{m-j} B \Gamma e^{[k]}(j) \\
& -C \sum_{j=0}^{m-1} \sum_{\mu=1}^{r} A^{m-j-1} E_{\mu} \\
& \times\left(x^{[k+1]}\left(j-h_{\mu}(j)\right)-x^{[k]}\left(j-h_{\mu}(j)\right)\right)
\end{aligned}
$$

Taking the norm of both sides of (20), and making use of the method employed above, we can obtain that for any $m \in \Gamma_{0}^{N}$,

$$
\begin{aligned}
\tilde{e}^{[k+1]}(t) \leq & \|I-C B \Gamma\| \tilde{e}^{[k]}(m) \\
& +\frac{\kappa a^{-\lambda}}{1-a^{-\lambda}}\left\|C\left(I-A^{-1}\right)\right\|\|B \Gamma\| \tilde{e}^{[k]}(m) \\
& +\frac{\kappa \delta a^{-\lambda}}{1-a^{-\lambda}}\left\|C A^{-1}\right\| \tilde{z}^{[k]}(m) \cdots(21)
\end{aligned}
$$

Furthermore, By substituting (19) into (21) we can have that for any $m \in \Gamma_{0}^{N}$,

$$
\begin{aligned}
& \tilde{e}^{[k+1]}(t) \leq(\|I-C B \Gamma\| \\
& +\frac{\kappa a^{-\lambda}}{1-a^{-\lambda}}\left\|C\left(I-A^{-1}\right)\right\|\|B \Gamma\| \\
& +\frac{\kappa \delta a^{-\lambda}}{1-a^{-\lambda}} \cdot \frac{1-a^{-\lambda}+\kappa a^{-\lambda}\left\|I-A^{-1}\right\|}{1-a^{-\lambda}-\kappa \delta a^{-\lambda}\left\|A^{-1}\right\|} \\
& \left.\times\left\|C A^{-1}\right\|\|B \Gamma\|\right) \tilde{e}^{[k]}(m)
\end{aligned}
$$

That is,

$$
\tilde{e}^{[k+1]}(m) \leq \rho(\lambda) \tilde{e}^{[k]}(m)
$$

where

$$
\rho(\lambda):=\|I-C B \Gamma\|+\eta(\lambda)
$$

and where

$$
\begin{aligned}
\eta(\lambda):= & \frac{\kappa a^{-\lambda}}{1-a^{-\lambda}}\left\|C\left(I-A^{-1}\right)\right\|\|B \Gamma\| \\
& +\frac{\kappa \delta a^{-\lambda}}{1-a^{-\lambda}} \cdot \frac{1-a^{-\lambda}+\kappa a^{-\lambda}\left\|I-A^{-1}\right\|}{1-a^{-\lambda}-\kappa \delta a^{-\lambda}\left\|A^{-1}\right\|} \\
& \times\left\|C A^{-1}\right\|\|B \Gamma\| \ldots \ldots \ldots \ldots \ldots \ldots(24)
\end{aligned}
$$

If the condition described by ( 8 ) is satisfied, it is obvious from (24) that there exists a positive constant $\lambda^{*}$ such that for any $\lambda \geq \lambda^{*}$ and for any $m \in \Gamma_{0}^{N}, \rho<1$. Therefore, we can obtain from (22) and the definition of $\tilde{e}^{[k]}(m)$ that for any $m \in \Gamma_{0}^{N}$,

$$
\lim _{k \rightarrow \infty}\left\|e^{[k]}(m)\right\|=0
$$

That is, the output error $e(m)$ converges asymptotically to zero, through the iterative learning process. Thus, we can complete the proof of this theorem.

Q.E.D. 
Remark 3.1 It is obvious from Assumption 2.1 that one can always choose an iterative learning control gain matrix $\Gamma$ for the considered discrete time-delay system such that the condition given in (8) is satisfied. Therefore, under Assumption 2.1, the existence of iterative learning control laws is well guaranteed. That is, we can always employ the iterative learning control schemes described by (5) with (6) such that the output error between the given desired output and the actual output converges asymptotically to zero, through the iterative learning process.

Remark 3.2 In this paper, we have employed a class of iterative learning control laws described by (5) and (6), which may regarded as the first-order updating laws. It is not difficult, from the method used in the proof of Theorem 3.1, that the result obtained here is extended to the problem of iterative learning control with high-order updating laws.

\section{An Illustrative Example}

To illustrate the utilization of our approach, in this section, we consider the following numerical example. Here, a discrete time-delay system is given in the following difference equation.

$$
\begin{aligned}
x(m+1)= & {\left[\begin{array}{ll}
0.3 & 0.1 \\
0.0 & 0.5
\end{array}\right] x(m)+\left[\begin{array}{l}
1 \\
1
\end{array}\right] u(m) } \\
& +\sum_{\mu=1}^{3} E_{\mu} x\left(m-h_{\mu}(m)\right) \cdots \cdots \cdots \\
y(m)= & {\left[\begin{array}{ll}
1 & 1
\end{array}\right] x(m) \ldots \ldots \ldots \ldots }
\end{aligned}
$$

where

$$
\begin{aligned}
& E_{1}=\left[\begin{array}{ll}
0.1 & 0.0 \\
0.1 & 0.1
\end{array}\right], \quad E_{2}=\left[\begin{array}{ll}
0.0 & 0.1 \\
0.1 & 0.0
\end{array}\right] \\
& E_{3}=\left[\begin{array}{ll}
0.1 & 0.0 \\
0.1 & 0.0
\end{array}\right]
\end{aligned}
$$

For the iterative learning control laws given in (5) and (6), in the light of the condition described by (8), we can select the iterative learning control gains as follows.

$$
\Gamma_{1}=0.6
$$

In addition, for iterative schemes (5) and (6), we give in this simulation the following initial conditions.

$$
\begin{aligned}
& u^{[0]}(m)=1.0, \quad m \in \Gamma_{0}^{N} \\
& \chi^{[0]}(m)=\left[\begin{array}{ll}
\cos (m) & \cos (m)
\end{array}\right]^{\top}, \quad m \in \Gamma_{0}
\end{aligned}
$$

Moreover, for simulation, we give the time delays $h_{\mu}(m), \mu=1,2,3$, as follows.

$$
\begin{aligned}
& h_{1}(m)= \begin{cases}1, & \text { if } m=2 n \\
0, & \text { if } m=2 n+1\end{cases} \\
& h_{2}(m)= \begin{cases}2, & \text { if } m=2 n \\
1, & \text { if } m=2 n+1\end{cases}
\end{aligned}
$$

$$
h_{3}(m)= \begin{cases}3, & \text { if } m=2 n \\ 2, & \text { if } m=2 n+1\end{cases}
$$

where $n=1,2, \ldots$.

For $(25)$, the desired output trajectory $y_{r}(m)$ is given as follows.

$$
y_{r}(m)=1-\cos (0.1 m) \cdot
$$

where $m \in \Gamma_{0}^{N}$ and $N=60$.

Here, let the final tracking error be defined as

$$
\psi(k):=\max _{m}\left\{\left|e^{[k]}(m)\right|, \quad m \in \Gamma_{0}^{N}\right\}
$$

and for the learning processes of initial state, we define

$$
\phi(k):=\left\|\chi^{[k]}(0)\right\|
$$

Then, the results of a simulation are shown in Fig.1 and Fig. 2 for this discrete time-delay system with the chosen parameter settings.

It can be observed from Fig.1 and Fig.2 that by using the proposed iterative learning control laws, we can guarantee the asymptotic convergence of the output error between the given desired output and the actual output for all $m \in \Gamma_{0}^{N}$.

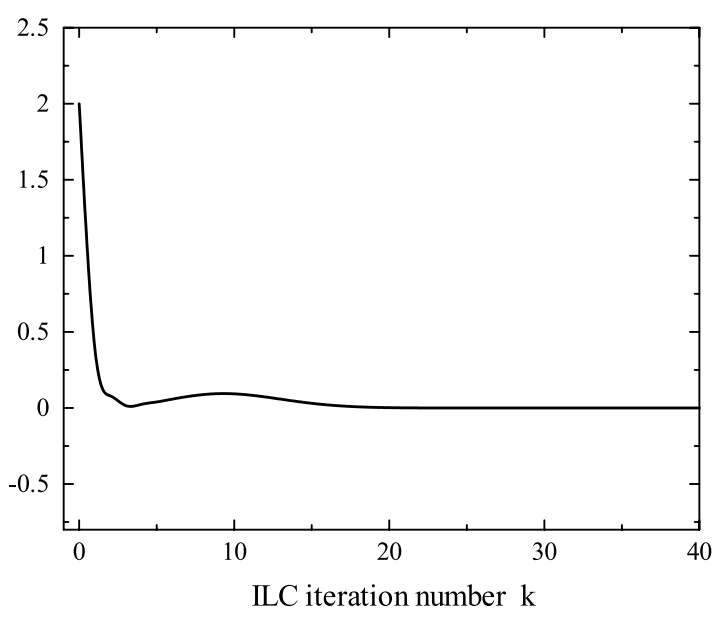

Fig. 1. The tracking error bound $\psi(k)$

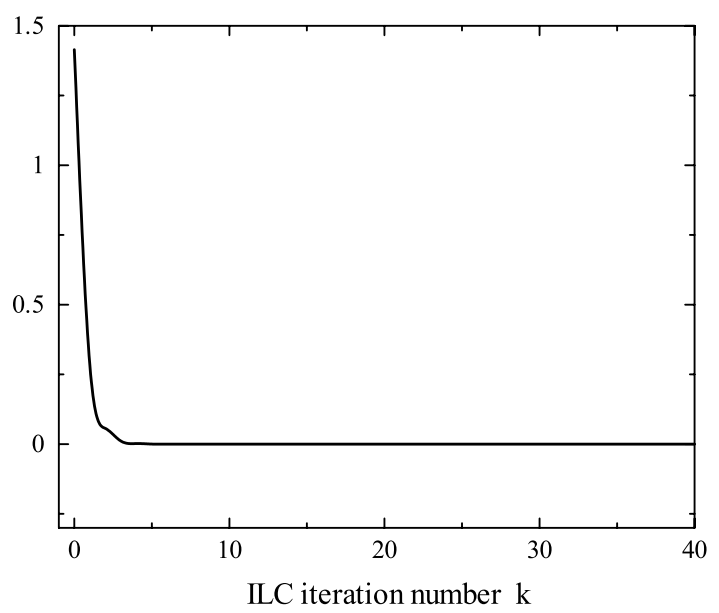

Fig. 2. The learning process of the initial state $\phi(k)$ 


\section{Concluding Remarks}

The problem of iterative learning control has been considered for linear discrete systems with time-delay. It has been assumed that the initial condition for discrete time-delay systems is unknown at each iteration. A method has been presented whereby a class of iterative learning control schemes can be constructed. It has also been shown that under given conditions, the constructed iterative learning controllers can guarantee the asymptotic convergence of the output error between the given desired output and the actual output through the iterative learning process. The method proposed in this paper can be extended to nonlinear discrete timedelay systems to construct some discrete iterative learning control algorithms.

Finally, a numerical example is given to demonstrate the synthesis procedure for the proposed iterative learning control schemes. It is shown from the example and the results of its simulation that the results obtained in the paper are effective and feasible. Therefore, our results may be expected to have some applications to practical iterative learning control problems of discrete time-delay systems.

(Manuscript received Oct. 4, 2004, revised Dec. 19, 2005)

\section{References}

(1) S. Arimoto, S. Kawamura, and F. Miyazaki: "Bettering Operation of Robots by Learning", J. Robotic Syst., Vol.1, pp.123140 (1984)

( 2 ) T. Sugie and T. Ono: "An Iterative Learning Control Law for Dynamical Systems", Automatica, Vol.27, pp.729-732 (1991)

( 3 ) N. Amann, D.H. Owens, and R. Eric: "Predictive Optimal Iterative Learning Control", Int. J. Contr., Vol.69, pp.203-226 (1998)

(4) H. Jay, K.S. Lee, and W.C. Kim: "Model Based Iterative Learning Control with a Quadratic Criterion for Linear TimeVarying Systems", Automatica, Vol.36, pp.641-657 (2000)

(5) G. Heininger, D. Fenwick, B. Paden, and F. Miyazaki: "Stability of Learning Control with Disturbances and Uncertain Initial Conditions", IEEE Trans. Automat. Contr., Vol.AC37, pp.110-114 (1992)

(6) C.Y. Wen, Y.Q. Chen, and M.X. Sun: "Robust High-Order P-Type Iterative Learning Controller Using Current Iteration Tracking Error", Int. J. Contr., Vol.68, pp.331-342 (1998)

(7) J. Ghosh and B. Paden: "A Pseudoinverse-Based Iterative Learning Control", IEEE Trans. Automat. Contr., Vol.AC-
47, pp.831-837 (2002)

( 8 ) T.J. Jang, C.H. Choi, and H.S. Ahn: "Iterative Learning Control in Feedback Systems", Automatica, Vol.31, pp.243-248 (1995)

( 9 ) Y. Chen, C. Wen, Z. Gong, and M. Sun: "An Iterative Learning Controller with Initial State Learning", IEEE Trans. Automat. Contr., Vol.AC-44, pp.371-376 (1999)

(10) K.H. Park, Z. Bien, and D.H. Hwang: "Design of an Iterative Learning Controller for a Class of Linear Dynamic Systems with Time Delay", IEE Proc. - Control Theory Appl., Vol.145, pp.507-512 (1998)

(11) Y. Chen, Z. Gong, and C. Wen: "Analysis of High-Order Iterative Learning Control Algorithm for Uncertain Nonlinear Systems with State Delays", Automatica, Vol.34, pp.345-353 (1998)

(12) Z. Geng, R.L. Carroll, and J. Xie: "Two-Dimensional Model Algorithm Analysis for a Class of Iterative Learning Control Systems", Int. J. Contr., Vol.52, pp.833-862 (1990)

(13) J.E. Kurek and M.B. Zaremba: "Iterative Learning Control Synthesis Based on 2-D System Theory", IEEE Trans. Automat. Contr., Vol.AC-38, pp.121-125 (1993)

(14) S.S. Saab: "A Discrete-Time Learning Control Algorithm for a Class of Linear-Invariant Systems", IEEE Trans. Automat. Contr., Vol.AC-40, pp.1138-1141 (1995)

(15) T. Ishihara, K. Abe, and T. Takeda: "A Discrete-Time Design of Robust Iterative Learning Controller", IEEE Trans. Syst. Man Cybernetics, Vol.22, pp.74-84 (1992)

Hansheng Wu (Member) received the B.S. and M.S. de-

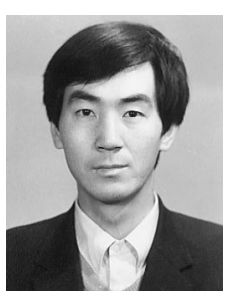
grees in Automatic Control Engineering from Northeast University of Technology, P.R. China in 1982 and 1984, respectively, and Ph.D. degree in Information Engineering from Hiroshima University, Japan in 1989. From November 1989 to September 1990, he was a Lecturer in the Department of Automatic Control, Northeast University of Technology, which was renamed Northeastern University in 1993. From October 1990 to March 1992, he was a Postdoctoral Fellow of the University of New South Wales, Australia in School of Mechanical and Manufacturing Engineering. From March 1992 to March 1993, he was a Fellow of Postdoctoral Fellowships for Foreign Researchers of Japan Society for the Promotion of Science (JSPS) at Hiroshima University. From April 1993 to March 1996, he was an Associate Professor in the Faculty of Integrated Arts and Sciences, Hiroshima University. Since April 1996, he has been with the Department of Information Science, Hiroshima Prefectural University, where he is currently a Professor.

His research interests include optimal control, dynamical games, large--scale systems, robust control, adaptive control, and their applications.

Dr. $\mathrm{Wu}$ is also a member of the Information Processing Society of Japan and the Society of Instrument and Control Engineers of Japan. 